\title{
Transport Spectroscopy of Chemical NANOSTRUCTURES: The Case of Metallic Single-Walled Carbon Nanotubes
}

\author{
Wenjie Liang, ${ }^{1}$ Marc Bockrath, ${ }^{2}$ and Hongkun Park ${ }^{1}$ \\ ${ }^{I}$ Department of Chemistry and Chemical Biology and Department of Physics, Harvard \\ University, Cambridge, Massachusetts 02138; email: HPark@chemistry.harvard.edu \\ ${ }^{2}$ Department of Applied Physics, California Institute of Technology, Pasadena, \\ California 91125
}

Key Words single-molecule transistor, Coulomb blockade, electronic shell filling, Kondo resonance, inelastic tunneling, electron wave interference

- Abstract Transport spectroscopy, a technique based on current-voltage measurements of individual nanostructures in a three-terminal transistor geometry, has emerged as a powerful new tool to investigate the electronic properties of chemically derived nanostructures. In this review, we discuss the utility of this approach using the recent studies of single-nanotube transistors as an example. Specifically, we discuss how transport measurements can be used to gain detailed insight into the electronic motion in metallic single-walled carbon nanotubes in several distinct regimes, depending on the coupling strength of the contacts to the nanotubes. Measurements of nanotube devices in these different conductance regimes have enabled a detailed analysis of the transport properties, including the experimental determination of all Hartree-Fock parameters that govern the electronic structure of metallic nanotubes and the demonstration of Fabry-Perot resonators based on the interference of electron waves.

\section{INTRODUCTION}

Optical spectroscopy has long served as the experimental tool of choice for investigating electronic and nuclear motions in atoms and molecules. In optical spectroscopy, the interaction between light and matter causes transitions between quantum states, and series of well-resolved spectral features are recorded as a function of the wavelength of light. The positions, intensities, and widths of the observed spectral features provide detailed information concerning the dynamics of electrons and nuclei in atoms and molecules. Optical spectroscopy has also been applied successfully to chemically derived nanostructures, such as nanocrystals (1-3), nanowires $(4,5)$, and carbon nanotubes $(6,7)$, providing insight into the effects of quantum confinement on the electronic properties of these nanostructures. 
Over the last decade, a new type of spectroscopy based on electron transport measurements, often termed transport spectroscopy, has emerged as a powerful tool for investigating electronic motions in chemical nanostructures. Originally developed for lithographically-defined quantum dots (8), transport spectroscopy is based on current-voltage measurements of a three-terminal transistorlike device in which a single chemical nanostructure bridges two electrodes, and a third gate electrode couples electrostatically to the nanostructure. The electronic conduction through such devices at sufficiently low temperatures is dictated by single-electron charging and energy level quantization (9). Plots of current as a function of bias voltage exhibit features that provide detailed information on quantum-level structures in chemical nanostructures that complements optical spectroscopy.

To date, transport spectroscopy has been carried out for a variety of chemical nanostructures, including molecules (10-14), nanocrystals (15-19), nanowires $(20,21)$, and single-walled carbon nanotubes (SWNTs) (22-34). Among these chemical nanostructures, SWNTs have served as a model system for transport studies. In addition to the radial electron confinement provided by their small diameter (35-38), the finite length of nanotubes serves to axially confine electrons. Hence, at sufficiently low temperatures, nanotubes act as one-dimensional quantum dots whose electronic properties can be probed via transport measurements.

In this review, we discuss recent experiments involving metallic SWNTs that demonstrate the power of transport spectroscopy. First, we review measurements in which the tunnel coupling between SWNTs and metallic electrodes is weak (22-25). The strength of the coupling between a SWNT and the attached electrodes is quantified by comparing the gate-voltage-averaged conductance $\left(G_{a v g}\right)$ of the nanotube device to the conductance quantum $G_{0}=e^{2} / h$ [the value of $e^{2} / h$ is $38.8^{\circ} \mu \mathrm{S}$ or $\left.(25.8 \mathrm{k} \Omega)^{-1}\right]$. When $G_{a v g} \ll G_{0}$, SWNT devices exhibit Coulomb blockade phenomena, and the study of their $I-V$ behavior provides evidence for energy-level quantization $(22,23)$ and spin filling $(24,25)$. As $G_{a v g}$ approaches $G_{0}$, additional transport features start to appear owing to the occurrence of higher-order tunneling phenomena, including Kondo resonance $(28,32)$, inelastic cotunneling $(28,32,39)$, and a striking four-electron periodicity in electron addition energy (32). The behavior of devices in this regime can be described by a shell-filling model that takes into account the two subbands present in nanotubes as well as the direct and exchange Coulomb interaction between the electrons, and allows for determination of all the Hartree-Fock parameters of nanotubes from the transport data alone (32). Finally, when $G_{a v g} \gg G_{0}$, the Coulomb blockade becomes suppressed and finite-length SWNTs act as resonant cavities for electron waves (30, 31, 33). The resulting devices exhibit conductance oscillations as the Fermi wavelength of the electrons is tuned by varying the gate voltage, thereby demonstrating ballistic and phase-coherent electron transport in nanotubes $(30,31,33)$. Combined together, the transport measurements of nanotube devices as a function of $G_{a v g}$ provide detailed insight into the electronic motion within SWNTs that is difficult to obtain through conventional optical spectroscopy. 


\section{NANOTUBE GROWTH AND DEVICE FABRICATION}

Transport spectroscopic investigation of SWNTs requires the fabrication of threeterminal devices, in which individual SWNTs function as the transistor channels. Because SWNTs can have lengths exceeding tens of microns, the device fabrication can be performed in a rather straightforward manner using either optical or electron-beam lithography, as illustrated in Figure 1 (see color insert). Here we briefly describe the device fabrication procedure $(29,30,32,40)$. Substrates for isolated nanotube growth are prepared from degenerately doped silicon wafers capped with a $1 \mu \mathrm{m} \mathrm{SiO}$ layer. A small piece of the wafer is dipped into a solution containing $22 \mathrm{mg} \mathrm{Fe}\left(\mathrm{NO}_{3}\right)_{3} \cdot 9 \mathrm{H}_{2} \mathrm{O}$ in $135 \mathrm{~mL}$ isopropanol, and then rinsed in hexane and dried. The wafer is placed in a quartz boat inside a 1-inch quartz tube furnace equipped with argon, hydrogen, and ethlyene gas sources. The furnace is purged by flowing 600 SCCM argon, 400 SCCM hydrogen, and 0.5 SCCM $\mathrm{C}_{2} \mathrm{H}_{4}$ for 5 minutes. The ethylene flow is reduced to zero, and the furnace is heated under argon and hydrogen to the reaction temperature $\left(700^{\circ} \mathrm{C}-800^{\circ} \mathrm{C}\right)$ in 20 minutes. Nanotubes are grown by turning on the ethylene flow at $0.5 \mathrm{SCCM}$ for $\sim 6$ minutes. Once the nanotube growth is complete, the furnace cools under argon. Atomic force microscopy (AFM) and transmission electron microscopy (TEM) studies indicate that this growth procedure results in predominantly individual SWNTs with diameter $\sim 1-3 \mathrm{~nm}$; small numbers of multiwalled nanotubes are of diameter greater than $2 \mathrm{~nm}$.

After the growth process, the wafers are inspected by AFM to identify wafers with a suitable density of isolated SWNTs. A resist bilayer of PMMA-MMA is then spun on the appropriate wafers, and alignment marks are defined using electron-beam lithography, followed by evaporation of chromium (Cr: $35 \AA$ thick) and gold (Au: $500 \AA$ thick). The remaining resist and unwanted metal are removed by lift-off in acetone. A thin layer of resist residue that typically remains after this procedure is then removed by heating the wafers to $250^{\circ} \mathrm{C}$ in flowing oxygen for 20 minutes. The wafers are inspected using an AFM to locate suitable $\sim 1-\mathrm{nm}$ diameter isolated SWNTs relative to the alignment marks, as shown in Figure $1 a$. Once the positions of $\sim 12$ such nanotubes are located, another resist bilayer is spun onto the wafers and the electrodes are defined by electron-beam lithography, followed by evaporation of $\mathrm{Cr}$ and $\mathrm{Au}$. Figure $1 b$ shows an AFM image of a typical device fabricated in this fashion.

\section{TRANSPORT SPECTROSCOPY OF SINGLE-WALLED CARBON NANOTUBES}

Figure 2 (see color insert) shows the resulting device geometry, consisting of the SWNT and the attached electrodes on the substrate. The degenerately doped substrate acts as a gate electrode, allowing the transport properties of the SWNTs to be studied as a function of charge density. Both semiconducting and metallic SWNT 
devices have been characterized by transport measurements at room temperature. Here we concentrate on metallic nanotube devices that have an approximately gate-voltage-independent conductance (35-38). Panels in Figure 2 present plots of differential conductance $(d I / d V)$ as a function of source-drain bias voltage $(V)$ and gate voltage $\left(V_{g}\right)$ obtained from six representative devices at a temperature of $1.5 \mathrm{~K}$. The bar graphs to the right of the $d I / d V$ data show $G_{a v g}$ for each device. We have explored devices with a wide spectrum of conductance, from $G_{\text {avg }} \sim$ $0.01 e^{2} / h$, corresponding to weak tunnel coupling, to $G_{\text {avg }} \sim 3 e^{2} / h$, corresponding to strong coupling.

\section{DEVICES WITH WEAK TUNNEL COUPLING: COULOMB BLOCKADE}

Figure $3 a$ (see color insert) shows an enlarged $d I / d V-V-V_{\mathrm{g}}$ plot of the data shown in the first panel in Figure 2, obtained from a device with $G_{a v g} \ll G_{0}$. It clearly shows diamond-shaped conductance-gap regions in the $V-V_{g}$ plane where the current flowing through the nanotube is essentially zero. This behavior is characteristic of Coulomb blockade phenomena $(8,9)$. For the devices with $G_{a v g} \ll G_{0}$, the charge on the nanotube quantum dot is a good quantum number, and current flow must occur via single-electron charging and discharging events. Within the conductancegap regions, fluctuations in the number of electrons on the dot are suppressed owing to the energy cost of changing the charge state of the dot by one electron. The conductance gap is a thus a direct manifestation of charge discreteness.

In the Coulomb blockade regime, the equilibrium charge on the nanotube quantum dot is the integer number that minimizes the total energy of the system. At the gate voltages where two successive charge states become energetically degenerate, current flow becomes possible for arbitrarily small $V$, leading to the peak in $d I / d V$ at zero bias. As the gate voltage is increased through each conductance peak, the number of electrons that are energetically stable increases by one. Each successive conductance-gap region thus corresponds to a different charge state of a nanotube quantum dot.

The above considerations are illustrated schematically in the panels included in Figure 3. These diagrams depict discrete quantum levels within the nanotube quantum dot, the Fermi level of the two electrodes, and the tunnel barriers isolating the nanotube. The filled discrete states in the dot are separated from the empty states by an energy gap of magnitude $E_{\max }=e^{2} / C+\Delta E$, where $C$ is the total capacitance of the nanotube quantum dot and $\Delta E$ is the quantum level spacing. The first term, which is known as the charging energy, arises from the electrostatic energy required to add an extra electron to the dot. At the point marked with a gray circle in Figure 3, the Fermi level lies between filled and empty quantum levels, and there is insufficient energy to change the number of electrons on the nanotube quantum dot at zero bias and temperature. Therefore the conductance is essentially zero in this situation.

The electrochemical potential of the nanotube can be tuned relative to the Fermi level of the electrodes using the gate voltage. When an energy level of the 
nanotube quantum dot is aligned with those of the electrodes, the energy cost for changing the number of electrons on the dot is zero, and transport can occur by electrons hopping on and off the nanotube. This corresponds to the vertices where two conductance-gap regions meet. When a large enough bias voltage is applied and the electron has sufficient energy to overcome the gap, current starts to flow owing to resonant tunneling through the ground state of the nanotube quantum dot, leading to a stepwise increase in current or a peak in $d I / d V$. This situation is indicated by the blue circle in Figure 3. When even higher voltage is applied, excited quantum levels become accessible for electron transport, leading to additional peaks in $d I / d V$, as indicated by the red circle in Figure 3. The voltage positions of these peaks shift linearly with $V_{g}$ owing to the changing electrostatic potential of the nanotube. The positions of these $d I / d V$ peaks (or more precisely the voltage values at which these peaks meet the conductance-gap region) therefore provide the energy level spacing between discrete quantum levels of the nanotube quantum dot. In summary, although the gap regions arise primarily from charge discreteness and Coulomb repulsion, the features outside the gap reflect the discrete quantum states arising from the finite size of the nanotube.

Through a detailed analysis of the transport data, both the charging energy $e^{2} / C$ and the mean level spacing $\Delta E$ can be directly measured for a given nanotube quantum $\operatorname{dot}(22,23)$. Typically, it is found that the ratio $\left(e^{2} / C\right) / \Delta E \sim 6$ for metallic SWNTs, independent of their length, with $\Delta E \sim 1 \mathrm{meV}$ for a 1- $\mu \mathrm{m}$-long nanotube. The magnitude of $e^{2} / C$ and $\Delta E$ can be estimated using elementary electrostatics and the band structure of nanotubes, respectively. These estimates agree with the observed value of $\left(e^{2} / C\right) / \Delta E$, with both $e^{2} / C$ and $\Delta E$ scaling inversely with length yielding a length-independent ratio. The agreement between experimental results and these estimates demonstrates explicitly the one-dimensional nature of the electronic states in metallic SWNTs $(22,23)$.

\section{DEVICES WITH INTERMEDIATE TUNNEL COUPLING: ELECTRONIC SHELL FILLING}

As the $G_{\text {avg }}$ increases, nanotube devices start to exhibit new conductance features that were absent in the devices with weak tunnel coupling, as shown in the middle panels of Figure 2. Figure 4 (see color insert) shows a plot of $d I / d V$ at $V=0$ (linear response conductance) as a function of $V_{g}$ obtained from a representative nanotube device with $G_{a v g} \sim e^{2} / h$. The well-defined conductance peaks in Figure 4 denote the addition of single electrons to the nanotube quantum dot as the dot's electrostatic potential is tuned by varying $V_{g}$, as in the case of weak-coupling devices ( 9 , 22, 23). Remarkably, the conductance peaks in Figure 4 form a repeating pattern where the peaks appear in clusters of four, indicating that the electron addition to the nanotube quantum dot exhibits a four-electron periodicity (32). This four-electron periodicity is observed in nearly every nanotube device with $G_{\text {avg }} \sim 1-2 e^{2} / h$, demonstrating its robustness with respect to device-to-device variation.

Quantitative information about this electron addition pattern can be extracted from two-dimensional $d I / d V-V-V_{g}$ plots, such as those in Figure 5 (see color insert). 
Specifically, Figure 5 shows three small conductance-gap regions, labeled 1, 2, and 3 , followed by a large conductance-gap region labeled 4 . This conductance-gap pattern repeats over a large $V_{g}$ range corresponding to the addition of more than 20 electrons. The energy $\Delta \mu(N)$ associated with the addition of the $N$-th electron to a nanotube dot can be obtained by measuring the bias voltage at the upper and lower vertices of the diamond-shaped conductance-gap region (9). Denoting the corresponding $V$ values for each region by $V_{1-4}$, the addition energies for the first three electrons are $e V_{1-3} \sim 4 \mathrm{meV}$, whereas that for the fourth electron is $e V_{4} \sim 8 \mathrm{meV}$.

The dark horizontal lines in Figure 5, which correspond to the $d I / d V$ peaks with $V_{g}$-independent bias voltage positions, are additional transport characteristics that are universally observed in nanotube devices with $G_{\text {avg }} \sim 1-2 e^{2} / h$. In regions 1 and 3, these dark lines always appear at $V=0$, whereas they appear at nonzero bias voltages in region 4 . In region 2 , the $V$ positions of the dark lines vary, depending on the specific four-electron cluster under consideration.

The $d I / d V$ features appearing at $V=0$ are a signature of the Kondo resonance observed previously in lithographically defined quantum dots (41-46) and nanotube bundles (28). Kondo resonance is a many-electron phenomenon that results from the exchange interaction between a localized spin and the conduction electrons in the metallic electrodes $(41,42)$, and it appears when the electronic state of a quantum dot has nonzero spin $(S)$. The appearance of Kondo resonances within regions 1 and 3 therefore indicates that the corresponding electronic ground states have $S>0$. The intermittent appearance of a Kondo resonance in region 2 suggests that the ground state $S$ value in that region varies with the number of electrons on the nanotube quantum dot.

The $V_{g}$-independent $d I / d V$ features at nonzero bias in Figure 5 suggest, on the other hand, the occurrence of high-order tunneling processes where one electron enters the nanotube while another electron simultaneously exits the nanotube, leaving the nanotube dot in an excited state $(28,32,39)$. In this inelastic cotunneling process, the bias voltage $V_{\text {in }}$ supplies the excitation energy. Because this excitation does not involve a change in the charge state of the dot, the associated feature in $d I / d V$ is independent of $V_{g}$, and the value $e V_{\text {in }}$ provides a direct measure of the excited state energy. Upon application of a magnetic field, the horizontal features in region 2 shift in their $V$ position and merge into one horizontal line at $V=0$, suggesting a close connection between inelastic cotunneling and Kondo resonance $(28,32,45,47)$. The horizontal features in region 4 , which are far removed from $V=0$, exhibit a broadening of their widths under a magnetic field.

All these observations, including the four-electron periodicity, Kondo resonance, and inelastic cotunneling, can be explained in a unified fashion using a shell-filling model that incorporates the band structure of metallic SWNTs as well as the exchange and Coulomb interactions between electrons (48-51). Previous theoretical $(52,53)$ and experimental $(22,23,25,28,53-57)$ studies have shown that the electronic properties of metallic SWNTs are determined by two spindegenerate subbands with linear dispersion, as shown schematically in the inset 


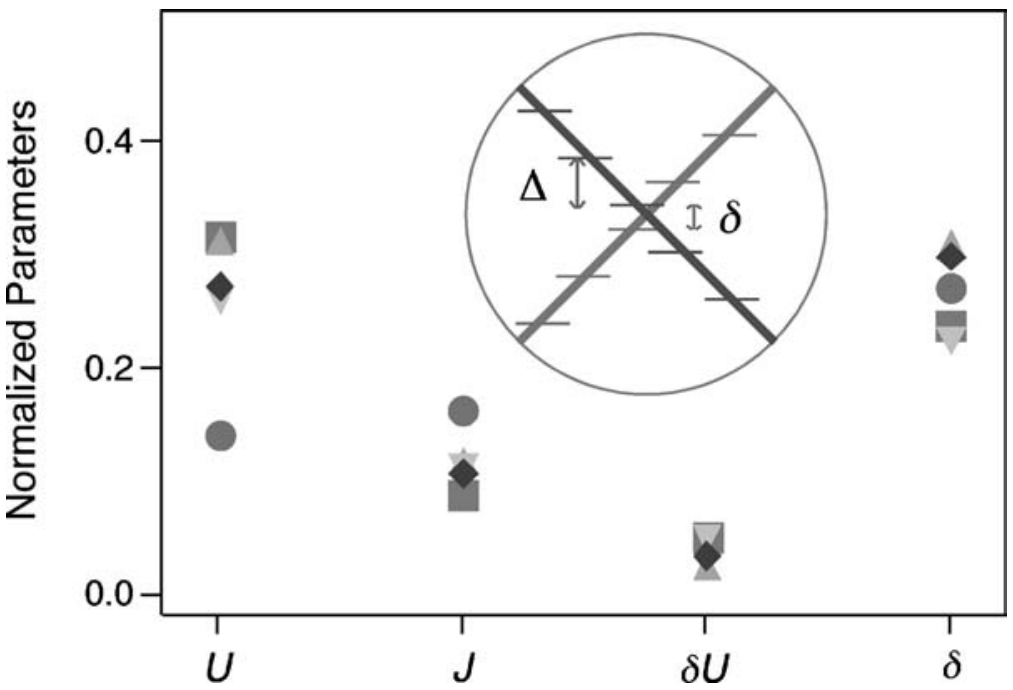

Figure 6 Values of $U, J, \delta U$, and $\delta$ normalized to $\Delta$ that are extracted from a nanotube device with a length of $200 \mathrm{~nm}$. Each shape indicates values obtained from a different four-electron cluster. The inset shows the single-particle-level structure appropriate for a nanotube quantum dot.

of Figure $6(30,32,52)$. In the simplest picture of a nanotube quantum dot, the quantization of these two subbands leads to two sets of spin-degenerate electronic levels with a mean spacing $\Delta$ within each set. The energy mismatch between these two sets of electronic levels can be represented by $\delta(\delta \leq \Delta / 2)$, as shown in the inset of Figure 6.

This picture can be further refined by including the Coulomb and exchange interactions, represented by three mean field parameters within the Hartree-Fock approximation: the charging energy $U$ that accounts for the long-range Coulomb repulsion between electrons, the exchange energy $J$ that favors spin alignment, and the excess Coulomb energy $\delta U$ to put two electrons into a single level (48). With these parameters, the Hamiltonian of the nanotube quantum dot can be written as (48)

$$
\begin{aligned}
H= & \sum_{\mu, \sigma, l} \varepsilon_{l \mu} n_{l \mu \sigma}+\frac{1}{2} U\left(\frac{Q_{d o t}-Q_{e x t}}{e}\right)^{2} \\
& +\delta U \sum_{\mu, l} n_{l \mu \uparrow} n_{l \mu \downarrow}+J \sum_{\mu, \mu^{\prime}} N_{\mu \uparrow} N_{\mu^{\prime} \downarrow}
\end{aligned}
$$

Here $\varepsilon_{l \mu}=l \Delta+(\mu-1) \delta$ is the energy of the $L$-th quantum level originating from the $\mu$-th subband ( $\mu=1,2$ ), $Q_{d o t}$ is the excess charge on the nanotube, $Q_{e x t}$ is the gate-induced charge on the dot, $n_{l \mu \sigma}$ is the number operator for the $L$-th level 
with spin $\sigma$ in the $\mu$-th subband, and $N_{\mu \sigma}$ is the total number of electrons with spin $\sigma$ in the $\mu$-th subband. Within this model, the five energy parameters, $\Delta, \delta$, $U, J$, and $\delta U$, completely determine the energies of all the possible spin-electronic configurations of a nanotube quantum dot (48).

This shell-filling model with two sets of spin-degenerate electronic levels provides a natural framework for the four-electron periodicity observed in Figures 4 and 5. Moreover, the shell-filling model explains the ground state spin configurations observed in Figure 5 as well. Assuming that $J$ and $\delta U$ are small compared to $\Delta$, two independent spin-filling schemes are possible in the model. In the first scenario, appropriate for $J+\delta U<\delta$, two electrons of opposite spin fill each spin-degenerate level before filling higher energy levels, producing the sequence of ground state spins $S=1 / 2, S=0, S=1 / 2$, and $S=0$. In the second scenario, in which $J+\delta U>\delta$, the ground state for two electrons is a spin triplet, and the sequence of ground state spins becomes $S=1 / 2, S=1, S=1 / 2$, and $S=0$. As discussed previously, Figure 5 shows that the ground states of a nanotube quantum dot in regions 1 and 3 are spin degenerate, whereas the electronic state in region 2 can be spin singlet or multiplet depending on the exact electron numbers. The experimental observation is therefore consistent with the spin-filling schemes outlined above, and indicates that both spin-filling scenarios are realized in a nanotube quantum dot. Moreover, the excited states responsible for the inelastic cotunneling features in region 2 and 4 can be accounted for by the triplet states, in a manner consistent with experimentally observed magnetic-field dependence.

The analysis of the experimental data further allows the quantitative determination of $\Delta, \delta, U, J$, and $\delta U$. This task is accomplished by equating the theoretically determined energies with their experimentally measured values and solving the resulting set of equations. In this analysis, $\Delta$ can be fixed using the experimentally determined nanotube length $(L)$ as $\Delta=h v_{F} / 2 L$, where $h$ is Plank's constant and $v_{F}$ is the Fermi velocity $(23,30)$.

Values of the energy parameters obtained from our analysis are presented in Figure 6. Here, the parameter values determined from five distinct four-electron periods of the same nanotube device remain approximately constant, although they do fluctuate as the electron number on the quantum dot is varied. These small fluctuations, which may be caused by disorder, account for the experimentally observed variation in ground state $S$ value in region 2 with the electron number. The averaged parameter values, normalized to $\Delta=h v_{F} / 2 L=8.4 \mathrm{meV}$, are given by $U / \Delta=0.26, J / \Delta=0.12, \delta U / \Delta=0.04$, and $\delta / \Delta=0.27$. Similar values for $J / \Delta \sim 0.1-0.2$ and $\delta U / \Delta \sim 0.05-0.1$ were found from other nanotube devices with $G_{\text {avg }} \sim 1-2 e^{2} / h$ as well, as shown in Figure 6 . These values for $J$ and $\delta U$ compare favorably with the theoretically predicted values for metallic armchair nanotubes of $J / \Delta=0.22$ and $\delta U / \Delta=0.11$ (48).

It is noteworthy that the value of $U / \Delta \sim 0.26$ determined from our analysis is an order of magnitude smaller than what is typically observed in nanotube dots with $G_{\text {avg }} \ll e^{2} / h$. This small value of $U$ is consistent with previous transport 
studies on semiconductor quantum dots with high conductance (58), and it plays a critical role for the clear observation of the transport features in Figure 5 and hence the determination of the relatively small energy scales such as $\delta U$, $J$, and $\delta$. The nonzero value of $\delta$ determined from our analysis suggests, on the other hand, that the subband degeneracy is broken in a nanotube quantum dot. The exact value of $\delta$ is expected to vary randomly in different nanotube devices depending on the boundary conditions at the nanotube-metal interface (48). Extensive analyses of the shell-filling model with $J / \Delta, \delta U / \Delta$ and $U / \Delta$ shown in Figure 6 indicate, however, that the four-electron periodicity should be preserved in approximately $70 \%$ of these nanotube devices, providing an explanation for the robust four-electron periodicity for nanotube devices with $G_{\text {avg }} \sim 1-2 e^{2} / h$.

\section{DEVICES WITH NEARLY TRANSPARENT CONTACTS: ELECTRONIC FABRY-PEROT RESONATORS}

When either or both tunnel junctions of a nanotube device have a conductance $G_{a v g}>G_{0}$, the charge on the dot is subject to quantum fluctuations. In this regime, the behavior of nanotube devices cannot be explained by the Coulomb blockade picture. We now turn to a detailed analysis of the transport data in this regime, concentrating on metallic nanotube devices with room-temperature resistances below $15 \mathrm{k} \Omega$ corresponding to $G_{\text {avg }}>2 G_{0}$. For these large conductance devices, charge discreteness is essentially lost, suppressing the Coulomb gap. Nevertheless, features arising from energy quantization are still retained, albeit significantly broadened in energy, as expected from the relatively short residence times of the electrons on the nanotube before escaping into the external electrodes.

Figure 7 shows a $d I / d V-V_{g}$ plot near $V=0$ obtained from a representative nanotube device at a temperature of $4.2 \mathrm{~K}$ (30). The length of the nanotube segment $(L)$ between two electrodes was $\sim 220 \mathrm{~nm}$ as determined by AFM. Below $\mathrm{T}=10 \mathrm{~K}$, the device exhibits pronounced $d I / d V$ oscillations that are quasiperiodic in $V_{g}$ with $G_{\text {avg }} \sim 3.2 e^{2} / h$. Figure 8 (see color insert) shows a twodimensional $d I / d V$ plot as a function of $V$ and $V_{g}$ obtained from a nanotube device with $L=\sim 530 \mathrm{~nm}$. The dips in $d I / d V$ appear as dark lines in Figure 8. The positions of the $d I / d V$ dips evolve smoothly as $V$ and $V_{g}$ change, forming a mesh of crisscrossing dark lines. The conductance behavior of a device with $G_{\text {avg }} \sim 2 G_{0^{-}}$ $3 G_{0}$ does not change substantially, as the temperature was varied from $4 \mathrm{~K}$ down to $100 \mathrm{mK}$.

Figures 7 and 8 illustrate several characteristics shared by all nanotube devices with $G_{\text {avg }}$ in the range of 2 to $3 e^{2} / h(30,31,33)$. In these devices, $d I / d V$ remains above $e^{2} / h$ irrespective of $V, V_{g}$, and T, indicating that the electrical behavior of these nanotube devices is distinct from those in the Coulomb-blockade regime $(22,23,25,28,29,32,35,53)$. In addition, the $d I / d V$ dips are typically more pronounced than $d I / d V$ peaks, as demonstrated by dominant dark lines in Figure 8. 


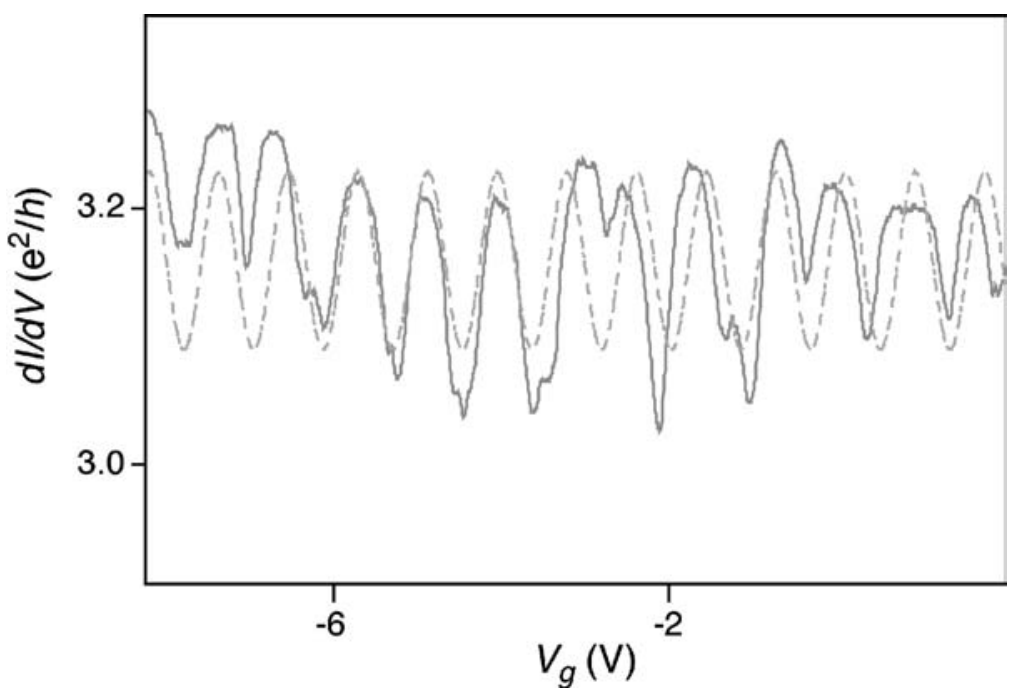

Figure 7 Plot of $d I / d V$ at $V=0$ as a function of $V_{g}$ obtained from a 220-nm-long nanotube device with $G_{\text {avg }} \sim 3.2 e^{2} / h$. The data are obtained at $\mathrm{T}=1.5 \mathrm{~K}$. The dotted curve shows a sinusoidal function with the same average period as the measured data. Comparison of these two plots demonstrates that the measured data is quasi-periodic in $V_{g}$.

Most importantly, the $V$ and $V_{g}$ spacing between adjacent dark lines increased as $L$ decreased. This last behavior is best quantified by the bias voltage $\left(V_{c}\right)$ at the crossing point between adjacent left- and right-sloped dark lines: Inspection of Figure 8 shows that $V_{c}$ for the $530-\mathrm{nm}$ device is $\sim 3.5 \mathrm{mV}$. The inset in Figure 8 shows the $V_{c}-L^{-1}$ plot obtained from seven different devices where $V_{c}$ can be unambiguously determined, and it clearly demonstrates the linear relationship between $V_{c}$ and $L^{-1}$.

As discussed previously, metallic SWNTs behave as one-dimensional ballistic conductors where the current is carried by two spin-degenerate one-dimensional transport modes with linear dispersion (Figures 6 and 9b) (22, 23, 25, 28, 52-57). The maximum $d I / d V$ value near-zero bias expected for a SWNT device is therefore $4 e^{2} / h$, obtained only when electrons pass through the nanotube-metal interfaces without reflection $(52,59)$. The mean conductance values in Figures 7 and 8 are smaller than this theoretical maximum, indicating the occurrence of electron scattering in the nanotube devices. The linear relationship between $V_{c}$ and $L^{-1}$ in the inset of Figure 8 nevertheless provides experimental evidence that the electron scattering occurs mostly at the nanotube-metal interface and that electrons pass through the nanotube ballistically (30).

This picture of interfacial electron scattering coupled with ballistic electron transport within the nanotube suggests that the nanotube device characteristics are 
(a)

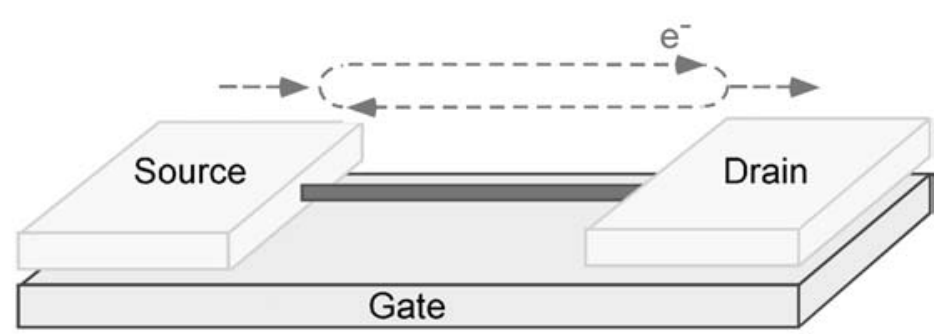

(b)

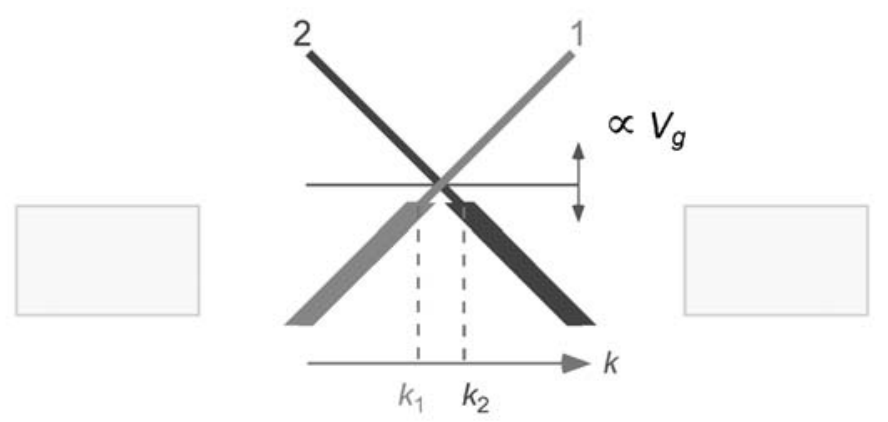

(c)

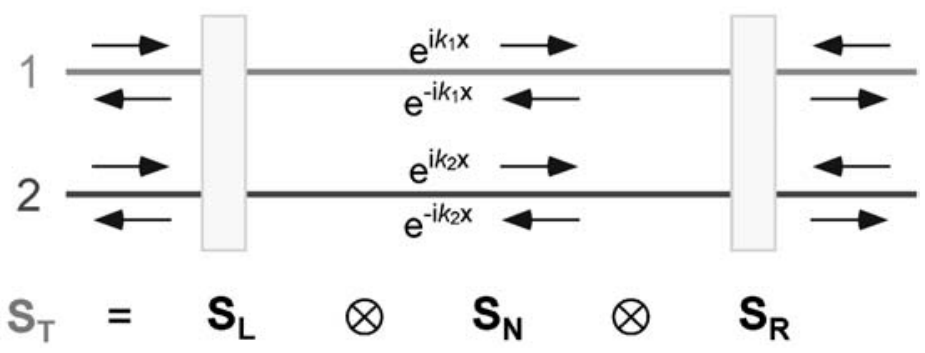

Figure 9 (a) Schematic diagram of a nanotube device with nearly transparent contacts. The diagram illustrates the multiple electron reflection that gives rise to the observed interference pattern. (b) Diagram of the band dispersion relation of a metallic single-walled carbon nanotube (SWNT) near a band-crossing point. In SWNTs, electronic states near two Fermi points located at $\mathbf{K}=\boldsymbol{k}_{0}$ and $\mathbf{K}^{\prime}=-\boldsymbol{k}_{0}$ contribute to the electrical conduction. The diagram shown here depicts only the band dispersion relation near $\mathbf{K}$ for clarity. The thick lines represent the electron filling in each band. The diagram shows the effect of $V_{g}$ on the electron filling in SWNTs when $V$ is zero. (c) Diagram that defines the $\mathbf{S}$ matrices $\mathbf{S}_{\mathbf{L}}, \mathbf{S}_{\mathbf{N}}, \mathbf{S}_{\mathbf{R}}$, and $\mathbf{S}_{\mathbf{T}}$. 
determined by quantum interference between electron waves multiply reflected between two nanotube-metal interfaces, analogous to light transmission in an optical Fabry-Perot cavity (60). Figures 7 and 8 show that $d I / d V$ oscillates as a function of $V_{g}$ and $V$, and the oscillation period exhibits an inverse dependence on the nanotube length. Because $V_{g}$ modulates the Fermi-level position in the nanotube and hence changes the Fermi wave number ( $2 \pi$ /wavelength) $k$ for electrons, the observed $d I / d V$ oscillations can be attributed to the change in $k$, just as in the case of transmitted intensity oscillations in an optical cavity.

The observed electron-wave interference can be understood quantitatively based on a theoretical model shown schematically in Figure 9. In this model based on the multichannel Landauer-Büttiker formalism (59, 61-63), the nanotube is considered a coherent electron waveguide with two propagating modes, and the electron scattering is modeled by $4 \times 4$ scattering (S) matrices at each interface, $\mathbf{S}_{\mathbf{L}}$ and $\mathbf{S}_{\mathbf{R}}$. Following previous theoretical studies $(52,53)$, the electron scattering between the two modes inside the nanotube is ignored, and the phase accumulation during electron propagation is represented by a diagonal $4 \times 4$ matrix, $\mathbf{S}_{\mathbf{N}}$. Transportmode coupling can still occur through the electron scattering at the interfaces, and the matrix elements of $\mathbf{S}_{\mathbf{L}}$ and $\mathbf{S}_{\mathbf{R}}$ represent not only the electron transmission and reflection within the same transport modes but also the transport-mode coupling (30).

One important difference between the nanotube electron cavity and a simple single-mode optical cavity arises from the fact that the two propagating modes in SWNTs are characterized by different wave vectors, $\boldsymbol{k}_{1}$ and $\boldsymbol{k}_{2}$, as shown in Figure $9 b(52,53)$. In an isolated neutral SWNT, $\boldsymbol{k}_{1}$ and $\boldsymbol{k}_{2}$ are the same and they are both given by $\boldsymbol{k}_{0}\left(k_{0}=8.44 \mathrm{~nm}^{-1}\right)$. In our nanotube devices, however, $\boldsymbol{k}_{1}$ and $\boldsymbol{k}_{2}$ are in general different owing to the linear band dispersion in SWNTs, and the difference between $\boldsymbol{k}_{1}$ and $\boldsymbol{k}_{2}$ grows linearly as the Fermi level is shifted by $V_{g}$. Consequently, electrons in the two propagating modes acquire different phase shifts as they traverse the nanotube, which are represented by the diagonal matrix elements of $\mathbf{S}_{\mathbf{N}}$. The phase change as a function of electron energy is responsible for the interference patterns as a function of $V$ and $V_{g}$ in Figures 7 and 8.

The overall conductance behavior of a nanotube device can be calculated once the device $\mathbf{S}$ matrix $\mathbf{S}_{\mathbf{T}}$ is obtained from $\mathbf{S}_{\mathbf{L}}, \mathbf{S}_{\mathbf{R}}$, and $\mathbf{S}_{\mathbf{N}}$ by matrix combination $(59,61-63)$. Specifically, in the zero-temperature limit, $d I / d V$ as a function of $V$ and $V_{g}$ in a nanotube device is related to the matrix elements of $\mathbf{S}_{\mathbf{T}}$ as $(30)$

$$
\begin{aligned}
\frac{d I}{d V}\left(V, V_{g}\right)= & \frac{2 e^{2}}{h}\left\lfloor\sum_{i . j=1,2}\left|t_{i l, j r}\left(\frac{e V}{2 \hbar v_{F}}+\frac{\pi}{4} \frac{C_{L} V_{g}}{e}\right)\right|^{2}\right. \\
& \left.+\sum_{i . j=1,2}\left|t_{i l, j r}\left(\frac{-e V}{2 \hbar v_{F}}+\frac{\pi}{4} \frac{C_{L} V_{g}}{e}\right)\right|^{2}\right\rfloor .
\end{aligned}
$$


In this formula, $t_{i l, j r}$ represents matrix elements of $\mathbf{S}_{\mathbf{T}}, v_{F}$ is the Fermi velocity inside the nanotube $\left(v_{F} \sim 8 \times 10^{5} \mathrm{~m} / \mathrm{s}\right)$, and $C_{L}$ is the capacitance per unit length of a nanotube. This formula specifies not only the linear-response behavior of a nanotube device but also its nonlinear response upon the application of a bias voltage. Although the above formula is derived for the zero-temperature limit, it can still be used to fit the data in Figure 8 because the observed conductance behavior did not change as the temperature was lowered from $4 \mathrm{~K}$ down to $100 \mathrm{mK}$. This observation indicates that thermal smearing played little role in determining the transport properties.

The result of the theoretical fit for the 220-nm nanotube device is presented in Figure 10 (see color insert) along with the experimental data. In this theoretical fit, the matrix elements of $\mathbf{S}_{\mathbf{L}}$ and $\mathbf{S}_{\mathbf{R}}$, which represent the transmission and reflection coefficients at each nanotube-metal interface including the phase shifts, are used as fitting parameters. Figure 10 clearly shows that the theoretical fit accounts for the major features in the data satisfactorily. Although they are not shown, the calculations reproduce experimental data from other devices as well. One of the most important successes of the model is that it explains the energy scale of the observed $d I / d V$ oscillations (represented by $e V_{c}$ ) without any adjustable parameters. As shown in Figure 9, two transport bands in SWNTs have $E$ - $k$ slopes with equal magnitude and opposite sign. This band structure guarantees that whenever the Fermilevel change causes a round-trip phase shift of $+2 \pi$ in one mode, the other mode acquires a phase shift of $-2 \pi$. The value of $V_{c}$ can therefore be obtained by setting the round-trip phase shift $2 L e V_{c} /\left(h v_{F}\right)$ equal to $2 \pi$. Figure 8 (inset) shows a solid line obtained from this expression along with experimental data, illustrating that the theoretical model explains experimental $V_{c}$ values very well without any adjustable parameters.

Inspection of the fitting parameters obtained from the theoretical model shows that the pronounced $d I / d V$ dips cannot be reproduced without the intermode coupling at the interface, especially when the average conductance of the device is above $3 e^{2} / h$ as in Figure 10. This observation strongly suggests that the transportmode coupling at the nanotube-metal interface is important in our device geometry. It should also be noted, however, that several features in the experimental data remain unexplained by the theoretical model. Most notably, Figure 7 shows that the magnitudes of the observed $d I / d V$ dips are not uniform and show variations on the order of $0.1 e^{2} / h$ as $V_{g}$ is varied. Moreover, Figure 8 shows that this variation leads to superstructures in the two-dimensional plot that are not periodic in $V_{g}$. These observations may indicate the possible effects of disorder on electron propagation in the nanotube. This disorder is likely to arise from the underlying substrate or at the interface because intrinsic nanotube defects change electron transport properties much more drastically (29). Figure 8 also shows that the magnitudes of $d I / d V$ modulations diminish as $|V|$ is increased. This observation suggests the occurrence of heating or dephasing when the electron energy distribution deviates from equilibrium. The dephasing may arise from the fact that the nanotube-metal interface is not abrupt and instead spans many atoms. 


\section{CONCLUSION}

We have utilized transport spectroscopy to study nanotube transistors with a wide range of conductance values, and have found the strength of the coupling to the leads has a profound influence on the device behavior. Low conductance devices with weak tunnel coupling display a Coulomb gap in their $I-V$ characteristics, consistent with single-electron tunneling. Detailed analysis of the data in this regime provides insight into the one-dimensional nature of the discrete electronic states in nanotubes.

Devices with an intermediate coupling strength exhibit a number of novel physical phenomena, including a fourfold periodicity for electron addition energies, Kondo resonance, and inelastic cotunneling. Analysis of transport data from devices in this regime provides information about the electronic states in nanotubes, including the Hartree-Fock parameters describing the exchange interaction in nanotubes - the first time such information has been obtained for a chemically derived nanostructure. These results demonstrate the utility of transport experiments in understanding the behavior of electrons confined in chemically derived nanostructures.

Finally, for devices with strong tunnel coupling, the discreteness of charge becomes lost owing to quantum fluctuation in the number of electrons on the dots, thereby suppressing the Coulomb gap. However, features arising from the interference of electron waves reflecting off the nanotube-metal interface give rise to conductance oscillations, analogous to the oscillations in transmission of light through a Fabry-Perot optical cavity as the wavelength of the incident light is tuned. These conductance oscillations provide a vivid demonstration of ballistic and phase-coherent transport in nanotubes.

\section{ACKNOWLEDGMENTS}

The results reviewed in this paper were partially based on our work with other collaborators: Michael Tinkham, Charles M. Lieber, Dolores Bozovic, and Jason Hafner. We also thank Mark Gudiksen and Kristin Maher for helpful discussions. We gratefully acknowledge the support from NSF, DARPA, the Packard Foundation, the Dreyfus Foundation, and Harvard University.

\section{The Annual Review of Physical Chemistry is online at http://physchem.annualreviews.org}

\section{LITERATURE CITED}

1. Alivisatos AP. 1996. Science 271:933-37

2. Empedocles S, Bawendi M. 1999. Acct. Chem. Res. 32:389-96

3. Hu J, Li L-S, Yang W, Manna L,
Wang L-W, Alivisatos AP. 2001. Science 292:2060-63

4. Duan XF, Huang Y, Cui Y, Wang JF, Lieber CM. 2001. Nature 409:66-69 
5. Gudiksen MS, Lauhon LJ, Wang J, Smith DC, Lieber CM. 2002. Nature 415:61720

6. O'Connell MJ, Bachilo SM, Huffman CB, Moore VC, Strano MS, et al. 2002. Science 297:593-96

7. Hartschuh A, Pedrosa HN, Novotny L, Krauss TD. 2003. Science 301:1354-56

8. Kouwenhoven LP, Marcus CM, McEuen PL, Tarucha S, Westervelt RM, Wingreen NS. 1997. In Mesoscopic Electron Transport, ed. LP Kouwenhoven, G Schön, LL Sohn, pp. 105-214. Dordrecht: Kluwer

9. Grabert H, Devoret MH. 1992. Single Charge Tunneling. New York: Plenum

10. Park H, Park J, Lim AKL, Anderson EH, Alivisatos AP, McEuen PL. 2000. Nature 407:57-60

11. Park J, Pasupathy AN, Goldsmith JI, Chang C, Yaish Y, et al. 2002. Nature 417:722-25

12. Liang W, Shores MP, Bockrath M, Long JR, Park H. 2002. Nature 417:725-29

13. Yu LH, Natelson D. 2003. Nano Lett. 4:7983

14. Pasupathy AN, Bialczak RC, Martinek J, Grose JE, Donev LAK, et al. 2004. Science 306:86-89

15. Black CT, Ralph DC, Tinkham M. 1996. Phys. Rev. Lett. 76:688-91

16. Klein DL, McEuen PL, Bowen Katari JE, Roth R, Alivisatos AP. 1996. Appl. Phys. Lett. 68:2574-76

17. Ralph DC, Black CT, Tinkham M. 1997. Phys. Rev. Lett. 78:4087-90

18. Klein DL, Roth R, Lim AKL, Alivisatos AP, McEuen PL. 1997. Nature 389:699701

19. Park H, Lim AKL, Alivisatos AP, Park J, McEuen PL. 1999. Appl. Phys. Lett. 75: 301-3

20. De Franceschi S, van Dam JA, Bakkers EPAM, Feiner LF, Gurevich L, Kouwenhoven LP. 2003. Appl. Phys. Lett. 83:34446

21. Bjork MT, Thelander C, Hansen AE, Jensen LE, Larsson MW, et al. 2004. Nano Lett. 4:1621-25

22. Tans SJ, Devoret MH, Dai H, Thess A,
Smalley RE, et al. 1997. Nature 386:47476

23. Bockrath M, Cobden DH, McEuen PL, Chopra NG, Zettl A, et al. 1997. Science 275:1922-25

24. Cobden DH, Bockrath M, McEuen PL, Rinzler AG, Smalley RE. 1998. Phys. Rev. Lett. 81:681-84

25. Tans SJ, Devoret MH, Groeneveld RJA, Dekker C. 1998. Nature 394:761-64

26. Yao Z, Postma HWC, Balents L, Dekker C. 1999. Nature 402:273-76

27. Bockrath M, Cobden DH, Lu J, Rinzler AG, Smalley RE, et al. 1999. Nature 397:598601

28. Nygard J, Cobden DH, Lindelof PE. 2000. Nature 408:342-46

29. Bockrath M, Liang W, Bozovic D, Hafner JH, Lieber CM, et al. 2001. Science 291: 283-85

30. Liang W, Bockrath M, Bozovic D, Hafner JH, Tinkham M, Park H. 2001. Nature 411: 665-69

31. Kong J, Yenilmez E, Tombler TW, Kim W, Dai HJ, et al. 2001. Phys. Rev. Lett. 87: 106801

32. Liang WJ, Bockrath M, Park H. 2002. Phys. Rev. Lett. 88:126801

33. Mann D, Javey A, Kong J, Wang Q, Dai H. 2003. Nano Lett. 3:1541-44

34. Jarillo-Herrero P, Sapmaz S, Dekker C, Kouwenhoven LP, Van der Zant HSJ. 2004. Nature 429:389-92

35. Dekker C. 1999. Phys. Today 52(5):2228

36. McEuen PL. 2000. Phys. World 13:31-36

37. McEuen PL, Fuhrer M, Park H. 2002. IEEE Trans. Nanotech. 1:78-85

38. Dai H. 2002. Acct. Chem. Res. 35:103544

39. De Franceschi S, Sasaki S, Elzerman JM, van der Wiel WG, Tarucha S, Kouwenhoven LP. 2001. Phys. Rev. Lett. 86:878-81

40. Hafner JH, Bronikowski MJ, Azamian BR, Nikolaev P, Rinzler AG, et al. 1998. Chem. Phys. Lett. 296:195-202

41. Glazman LI, Raikh ME. 1988. JETP Lett. 47:452-55 
42. Meir Y, Wingreen NS, Lee PA. 1993. Phys. Rev. Lett. 70:2601-4

43. Goldhaber-Gordon D, Shtrikman H, Mahalu D, Abusch-Magder D, Meirav U, Kastner MA. 1998. Nature 391:156-59

44. Cronenwett SM, Oosterkamp TH, Kouwenhoven LP. 1998. Science 281:540 44

45. Sasaki S, De Franceschi S, Elzerman JM, van der Wiel WG, Eto M, et al. 2000. $\mathrm{Na}$ ture 405:764-67

46. van der Wiel WG, De Franceschi S, Fujisawa T, Elzerman JM, Tarucha S, Kouwenhoven LP. 2000. Science 289:21058

47. Pustilnik M, Avishai Y, Kikoin K. 2000. Phys. Rev. Lett. 84:1756-59

48. Oreg Y, Byczuk K, Halperin BI. 2000. Phys. Rev. Lett. 85:365-68

49. Tarucha S, Austing DG, Honda T, van der Hage RJ, Kouwenhoven LP. 1996. Phys. Rev. Lett. 77:3613-16

50. Kouwenhoven LP, Oosterkamp TH, Danoesastro MWS, Eto M, Austing DG, et al. 1997. Science 278:1788-92

51. Tarucha S, Austing DG, Tokura Y, van der Wiel WG, Kouwenhoven LP. 2000. Phys. Rev. Lett. 84:2485-88
52. White CT, Todorov TN. 1998. Nature 393:240-42

53. McEuen PL, Bockrath M, Cobden DH, Yoon Y-G, Louie SG. 1999. Phys. Rev. Lett. 83:5098-101

54. Frank S, Poncharal P, Wang ZL, De Heer WA. 1998. Science 280:1744-46

55. Venema LC, Wildoer WG, Janssen JW, Tans SJ, Tuinstra HLJT, et al. 1999. Science 283:52-55

56. Bachtold A, Strunk C, Salvetat J-P, Bonard J-M, Forró L, et al. 1999. Nature 397:67375

57. Tsukagoshi K, Alphenaar BW, Ago H. 1999. Nature 401:572-74

58. Molenkamp LW, Flensberg K, Kemerink M. 1995. Phys. Rev. Lett. 75:4282-85

59. Datta S. 1995. Electronic Transport in Mesoscopic Systems. Cambridge, UK: Cambridge Univ. Press

60. Hecht E. 1987. Optics. Reading: AddisonWesley

61. Büttiker M, Imry Y, Landauer R, Pinhas S. 1985. Phys. Rev. B 31:6207-15

62. Büttiker M. 1986. Phys. Rev. B 33:302026

63. Cahay M, McLennan M, Datta S. 1988. Phys. Rev. B 37:10125-36 
(a)

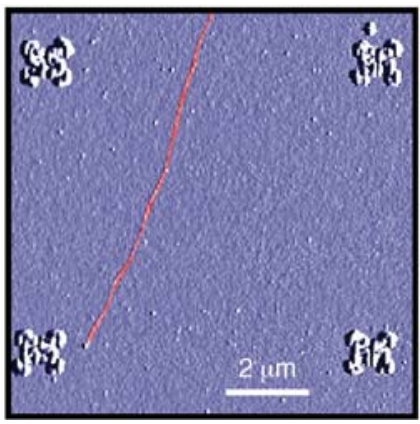

(b)

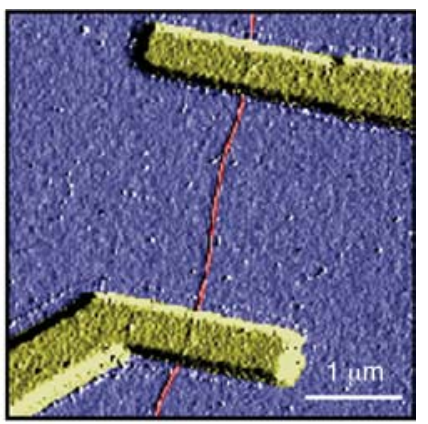

Figure 1 (a) Atomic force microscopy (AFM) image (false color) of a single-walled carbon nanotube grown on a degenerately doped silicon substrate. Gold alignment marks defined by electron-beam lithography are also shown in the image. (b) AFM image (false color) of a nanotube device. Metallic electrodes contacting the nanotube are defined by electron-beam lithography.

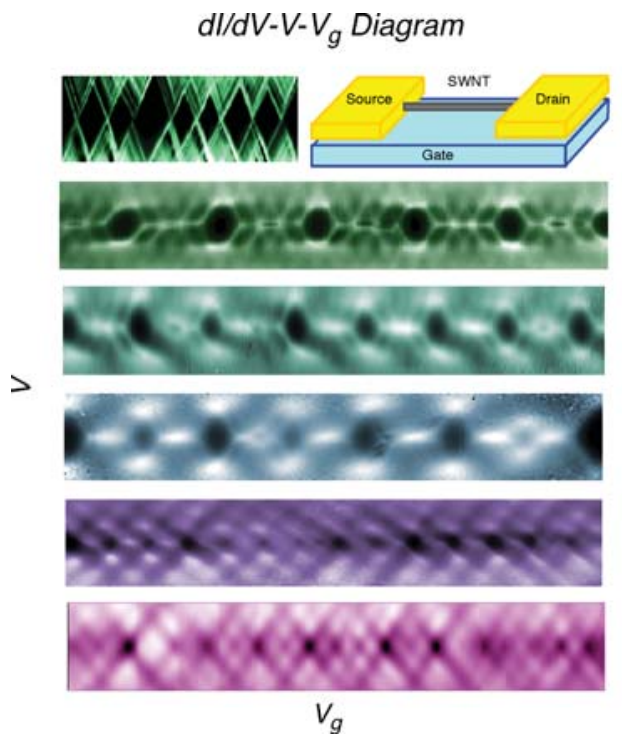

\section{Conductance}
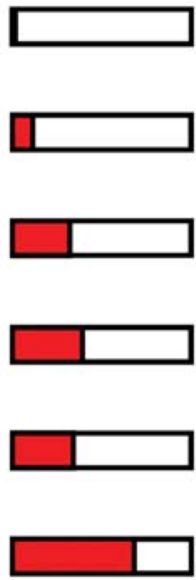

0

$4 \mathrm{e}^{2} / \mathrm{h}$

Figure 2 Plots of $d I / d V$ as a function of $V$ and $V_{g}$ obtained from six representative devices at a temperature of $1.5 \mathrm{~K}$. Bright colors represent higher conductance regions in every plot. The bar graphs on the right show the $V_{g}$-averaged conductance of each device. The inset shows a schematic diagram of a nanotube device. 

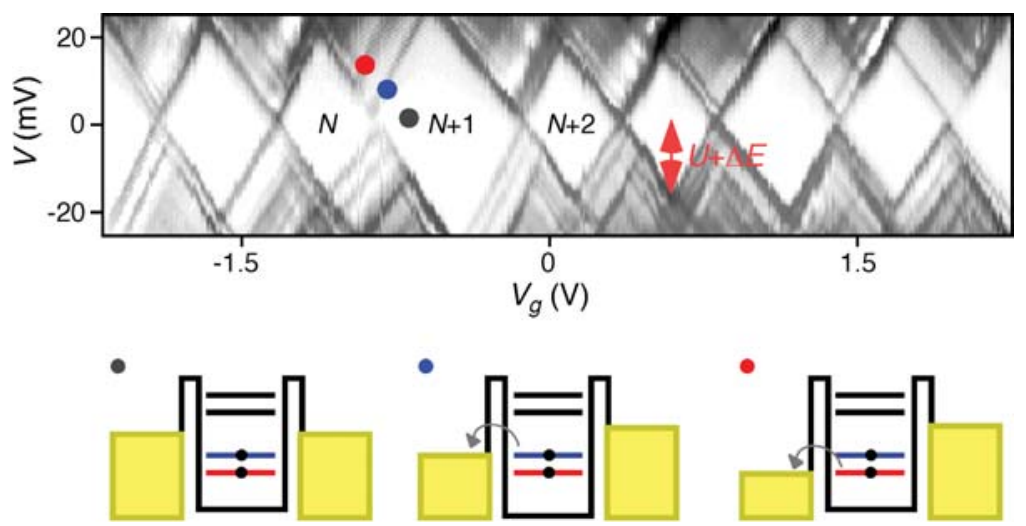

Figure 3 Plot of $d I / d V$ as a function of $V$ and $V_{g}$ obtained from a nanotube device with $G<<G_{0}$ at $\mathrm{T}=1.5 \mathrm{~K}$. Here, white color corresponds to 0 , and the darkest color corresponds to $0.5 e^{2} / h$. The underlying diagrams illustrate the alignments between the discrete quantum levels in a nanotube dot and the metal Fermi level at the $V$ - $V_{g}$ position denoted by each colored circle. In the figure, $\mathrm{U}$ represents the charging energy $\left(\mathrm{U}=e^{2} / \mathrm{C}\right)$, and $\Delta E$ represent the quantum level spacing.

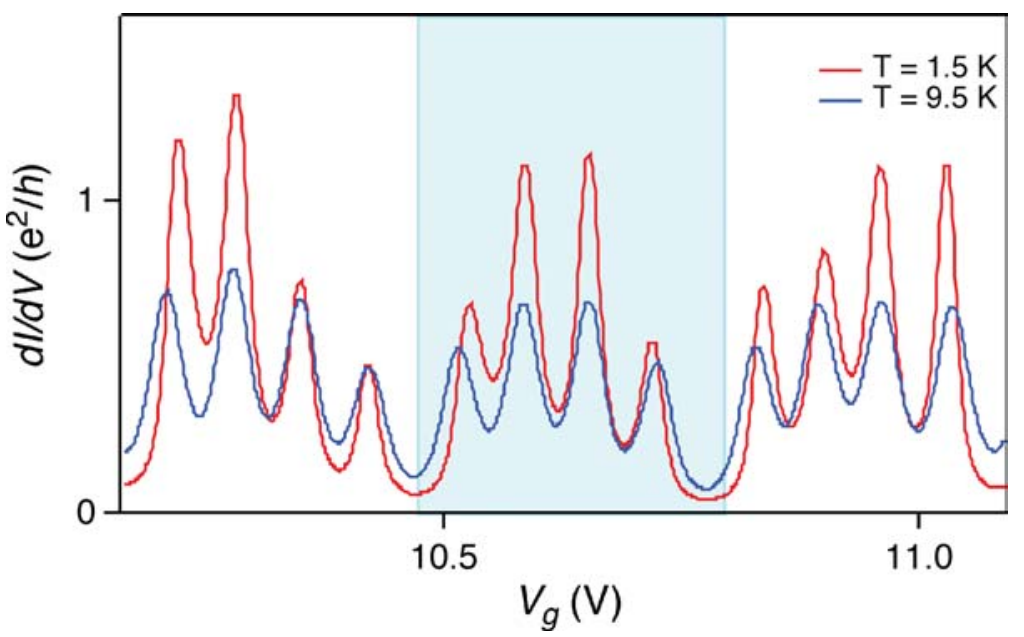

Figure 4 Plot of $d I / d V$ at $V=0$ as a function of $V_{g}$ obtained from a nanotube device with $G_{a v g} \sim e^{2} / h$. Red and blue traces are obtained at $\mathrm{T}=1.5 \mathrm{~K}$ and $9.5 \mathrm{~K}$, respectively. The shaded square denotes a cluster of four conductance peaks. This repeating feature is universally observed in nanotube devices with $G_{a v g} \sim e^{2} / h$. 
(a)

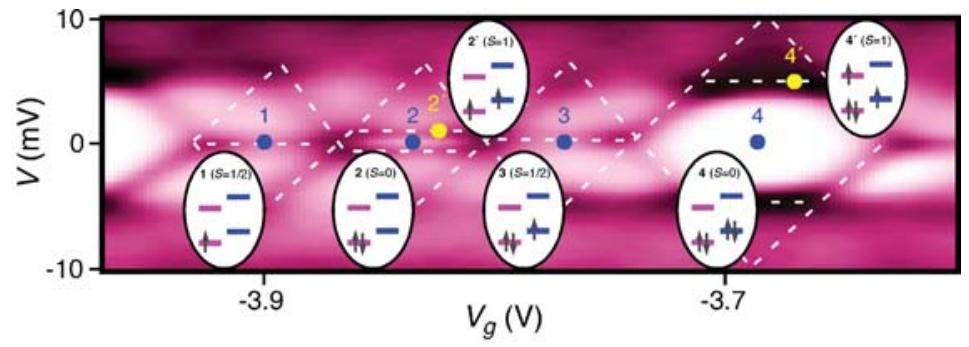

(b)

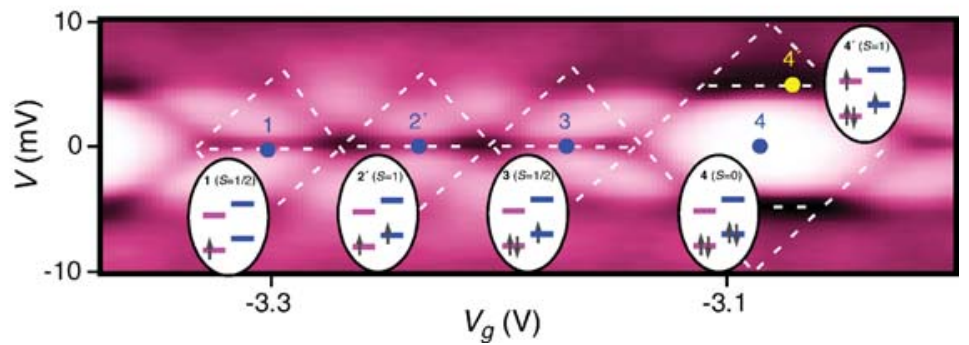

Figure 5 (a) Plot of $d I / d V$ as a function of $V$ and $V_{g}$ obtained from a nanotube device with $G_{a v g} \sim e^{2} / h$ at $\mathrm{T}=1.5 \mathrm{~K}$. Here, the white color corresponds to 0 , and the darkest color corresponds to $1.5 e^{2} / h$. The sloped white dashed lines delineate the conductance-gap regions that are marked by 1,2,3, and 4. (b) The same as in $(a)$, but for a different region of $V_{g}$. In both $(a)$ and $(b)$, the diagrams show the electronic configurations at the positions marked by numbered dots. Blue and yellow dots indicate the transport features arising from the ground and excited electronic states of the nanotube quantum dot, respectively.

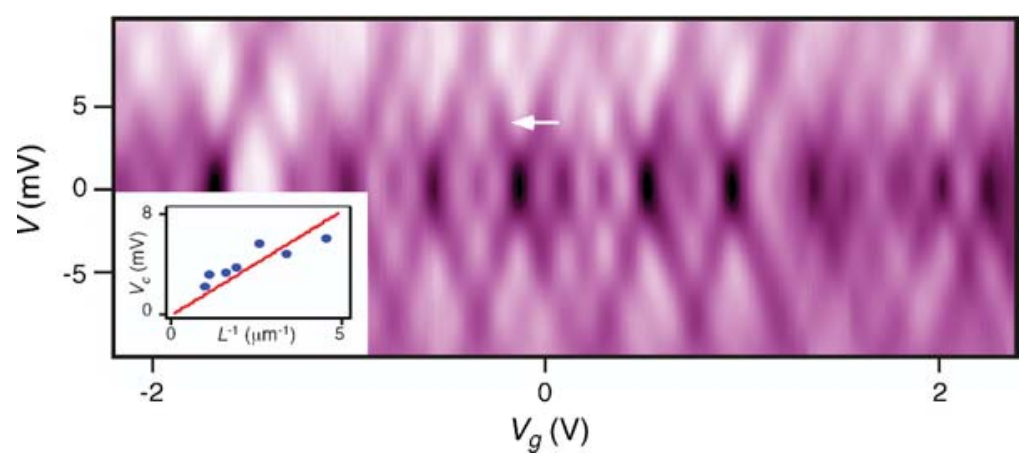

Figure 8 Plot of $d I / d V$ as a function of $V$ and $V g$ obtained from a 530-nm-long nanotube device with nearly transparent contacts. Here dark color corresponds to $1.5 e^{2} / h$, and white color corresponds to $3.3 e^{2} / h$. The data are obtained at $\mathrm{T}=4 \mathrm{~K}$. The white arrow marks the bias voltage value $\left(V_{c}\right)$ at which adjacent positively and negatively sloped lines intersect. The inset shows the values of $V_{c}$ obtained from 7 devices plotted against the inverse nanotube length $\left(L^{-1}\right)$. 


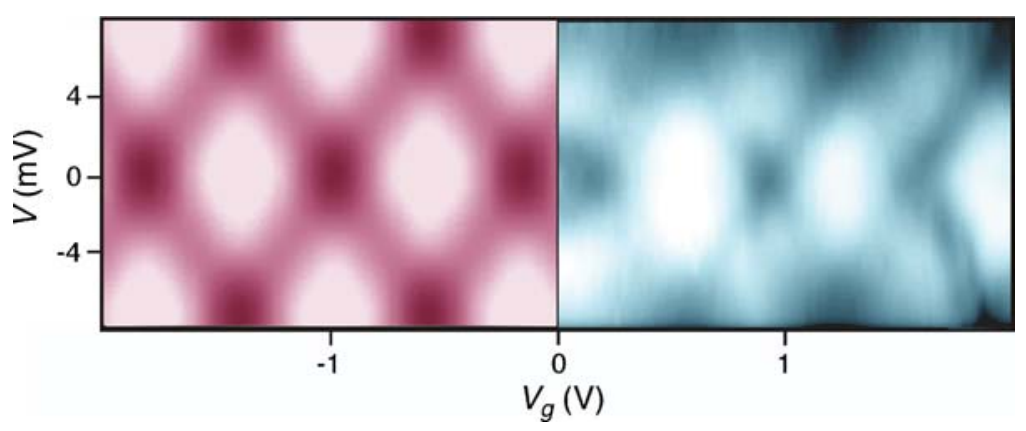

Figure 10 Calculated (left, shown in red) and measured (right, shown in blue) $d I / d V$ plots as a function of $V$ and $V_{g}$ for a 220-nm SWNT device. These plots have been shifted in $V_{g}$ for easy comparison. In both plots, dark color corresponds to $2.9 e^{2} / h$ and white color corresponds to $3.2 e^{2} / h$. 
争

Volume 56, 2005

\section{CONTENTS}

Quantum Chaos Meets Coherent Control, Jiangbin Gong and Paul Brumer

Femtosecond LASER PHOtOELECTRON SPECTROSCOPY ON ATOMS AND SMALl MoleCUles: PROTOTYPE STUdIES IN QUANTUM CONTROL, M. Wollenhaupt, V. Engel, and T. Baumert

NONSTATISTICAL DYNAMICS IN THERMAL REACTIONS OF POLYATOMIC MOLECULES, Barry K. Carpenter

RYDBERG WAVEPACKETS IN MOLECULES: FROM OBSERVATION TO CONTROL, H.H. Fielding

ELECTRON INJECTION AT DYE-SENSITIZED SEMICONDUCTOR ELECTRODES, David F. Watson and Gerald J. Meyer

QUANTUM MODE-COUPLING THEORY: FORMULATION AND APPLICATIONS TO NORMAL AND SUPERCOOLED QUANTUM LIQUIDS, Eran Rabani and David R. Reichman

Quantum MeChaniCs of DissiPATIVE Systems, YiJing Yan and RuiXue Xu

Probing Transient Molecular Structures in Photochemical PROCESSES USING LASER-INITIATED TIME-RESOLVED X-RAY ABSORPTION SPECTROSCOPY, Lin X. Chen

SEMICLASSiCAl INITIAL VALUE TREATMENTS of ATOMS AND MOLECULES, Kenneth G. Kay

Vibrational Autoionization in Polyatomic Molecules, S.T. Pratt

Detecting Microdomains In InTACT CELl Membranes, B. Christoffer Lagerholm, Gabriel E. Weinreb, Ken Jacobson, and Nancy L. Thompson

UltRAFAST CHEMISTRY: USING TIME-RESOLVED VIBRATIONAL SPECTROSCOPY FOR INTERROGATION OF STRUCTURAL DYNAMICS, Erik T.J. Nibbering, Henk Fidder, and Ehud Pines

MicROFLUIDIC TOOLS FOR STUDYING THE SPECIFIC BINDING, ADSORPTION, AND DISPLACEMENT OF PROTEINS AT INTERFACES, Matthew A. Holden and Paul S. Cremer 
AB InITIO QUANTUM CHEMICAL AND MiXed QuANTUM MECHANICS/MOLECULAR MECHANICS (QM/MM) METHODS FOR STUDYING ENZYMATIC CATALYSIS, Richard A. Friesner and Victor Guallar

FOURIER TRANSFORM INFRARED VIBRATIONAL SPECTROSCOPIC IMAGING: INTEGRATING MICROSCOPY AND MOLECULAR RECOGNITION, Ira W. Levin and Rohit Bhargava

TRANSPORT SPECTROSCOPY OF CHEMICAL NANOSTRUCTURES: The Case of Metallic Single-Walled Carbon Nanotubes, Wenjie Liang, Marc Bockrath, and Hongkun Park

ULTRAFAST ELECTRON TRANSFER AT THE MOLECULE-SEMICONDUCTOR NANOPARTICLE INTERFACE, Neil A. Anderson and Tianquan Lian

Heat Capacity in Proteins, Ninad V. Prabhu and Kim A. Sharp

METAL TO INSULATOR TRANSITIONS IN CluSTERS, Bernd von Issendorff and Ori Cheshnovsky

TIME-RESOLVED SPECTROSCOPY OF ORGANIC DENDRIMERS AND BRANCHED CHROMOPHORES, T. Goodson III

\section{INDEXES}

Subject Index

Cumulative Index of Contributing Authors, Volumes 52-56

Cumulative Index of Chapter Titles, Volumes 52-56

\section{ERRATA}

An online log of corrections to Annual Review of Physical Chemistry chapters may be found at http://physchem.annualreviews.org/errata.shtml 